TRANSACTIONS OF THE

AMERICAN MATHEMATICAL SOCIETY

Volume 362, Number 9, September 2010, Pages 4901-4920

S 0002-9947(10)05097-X

Article electronically published on April 9, 2010

\title{
MODULAR FORMS ON NON-LINEAR DOUBLE COVERS OF ALGEBRAIC GROUPS
}

\author{
HUNG YEAN LOKE AND GORDAN SAVIN
}

\begin{abstract}
We construct automorphic representations of non-linear two-fold covers of simply connected Chevalley groups via residues of Eisenstein series. In the process, we establish some basic results in representation theory of local groups.
\end{abstract}

\section{INTRODUCTION}

Let $\underline{G}$ be a split, simply connected algebraic group corresponding to an irreducible root system $\Phi$. The group $G$ can be constructed as a Chevalley group, which is defined over $\mathbb{Z}$. Over a local field $\mathbb{R}, \mathbb{Q}_{p}$ or the ring of adeles $\mathbb{A}=\mathbb{A}_{\mathbb{Q}}$, the group $\underline{G}$ has a unique non-trivial 2 -fold central extension denoted by $G$ :

$$
1 \rightarrow \mu_{2} \rightarrow G \rightarrow \underline{G} \rightarrow 1 \text {. }
$$

An irreducible representation of $G$ (local or global) is called genuine if the central subgroup $\mu_{2}$ acts via the unique non-trivial character on the representation. The central extension $G(\mathbb{A})$ splits over the group of rational points $\underline{G}(\mathbb{Q})$. Thus it is natural to study the space $L_{\text {gen }}^{2}(\underline{G}(\mathbb{Q}) \backslash G(\mathbb{A}))$ where the subscript gen indicates that we consider only the functions $f$ such that $f(\epsilon g)=\epsilon f(g)$ for every $\epsilon$ in $\mu_{2}$. This problem is a natural continuation of the study of classical modular forms of half integral weight. One purpose of this paper is to define Eisenstein series on $G(\mathbb{A})$ and to construct residual representation(s) $\Theta$ which, if $\underline{G}=\mathrm{SL}_{2}$, correspond to the classical theta series $1+2 \sum_{n>0} q^{n^{2}}$ or its anti-holomorphic analogue. Along the way, we study principal series representations of groups $G\left(\mathbb{Q}_{p}\right)$ where $p$ is any prime.

In order to explain our results here, let $\underline{T}$ be a maximal split torus in $\underline{G}$. Then its inverse image $T$ in $G$ is not necessarily commutative. Since the Weyl group acts by conjugation on irreducible genuine representations of $T\left(\mathbb{Q}_{p}\right)$, a natural question is whether there are Weyl group-invariant representations. A need for such representations is obvious: If $V$ is a genuine representation of $T\left(\mathbb{Q}_{p}\right)$, then we can define a family of representations $i(\chi)=V \otimes \chi$ by twisting with unramified characters of the torus $\underline{T}\left(\mathbb{Q}_{p}\right)$. If $V$ is Weyl group-invariant, then the conjugation action of the Weyl group on $i(\chi)$ reduces to the conjugation action on the character $\chi$. In this way, at least, one can express some basic results on principal series in a neat way. For example, if $G=\mathrm{Sp}_{2 n}$, then Weyl group invariant $V$ can be constructed using the Weil index W] [Rao. On the other hand, in Sa2 an explicit construction of

Received by the editors October 15, 2008 and, in revised form, April 9, 2009.

2010 Mathematics Subject Classification. Primary 22E46, 22E47.

(C)2010 American Mathematical Society 
such representations is given for simply laced groups. However, the corresponding Weyl group invariance was obtained by a somewhat tedious check using relations in the Steinberg group. In this paper we present a more natural construction of those representations of $T\left(\mathbb{Q}_{p}\right)$. Their Weyl group invariance will follow from a simple global argument. More precisely, our result is based on an observation that the analogous problem for real groups already has a solution for real groups, as given by Adams, Barbasch, Paul, Vogan and Trapa in A-V. Let $K_{\infty}$ be a maximal compact subgroup of $G(\mathbb{R})$. Recall that $T(\mathbb{R})$ has a decomposition $M A$, where $M$ is the centralizer of $A$ in $K_{\infty}$. The group $K_{\infty}$ has certain small genuine representations, called pseudo-spherical representations, whose property is that they reduce irreducibly to $M$. In particular, Weyl group invariance of such representations of $M$ is now obvious. Next, we consider the space

$$
L_{\text {gen }}^{2}(A \underline{T}(\mathbb{Q}) \backslash T(\mathbb{A}))
$$

of automorphic representations of $T(\mathbb{A})$. Given a pseudo-spherical type $\delta$, one easily sees that there is only one automorphic representation $\pi=\bigotimes \pi_{v}$ of $T(\mathbb{A})$ such that $\pi_{\infty} \cong \delta$ and $\pi_{p}$ is unramified for all primes $p$. The uniqueness of $\pi$ and the Weyl group invariance of $\delta$ immediately imply the Weyl group invariance of all $\pi_{p}$. If $\underline{G}=\mathrm{Sp}_{2 n}$, then one easily sees that our construction gives a Weil index.

We use $\pi$ to define local principal series representations, the corresponding Eisenstein series and a global residual representation $\Theta$ of Eisenstein series. Moreover, if $p \neq 2$ we use the central character $\gamma_{p}$ of $\pi_{p}$ to normalize Hecke operators in the Iwahori Hecke algebra $\mathcal{H}_{-}$of $G(\mathbb{Q})$. Following $[\mathrm{Sa2}$ this Hecke algebra is isomorphic to the Iwahori Hecke algebra of an algebraic group $\underline{G}^{l}$. This isomorphism allows us to (Shimura) lift genuine representations of $G\left(\mathbb{Q}_{p}\right)$ with Iwahori fixed vectors to the linear group $\underline{G}^{l}\left(\mathbb{Q}_{p}\right)$. We show that the Shimura lift sends unitary representations to unitary representations. For example, the local component $\Theta_{p}$ of $\Theta$ lifts to the trivial representation of $\underline{G}^{l}\left(\mathbb{Q}_{p}\right)$. In particular, if $\underline{G} \neq \mathrm{SL}_{2}$, it follows that $\Theta_{p}$ is isolated in the unitary dual of $G\left(\mathbb{Q}_{p}\right)$. We emphasize once again that the representation $\Theta$ and the isomorphism of Hecke algebra depend on the choice of the pseudo-spherical type $\delta$.

\section{AN AdÈLIC Group}

Let $\Phi$ be a root system with simple roots $\triangle=\left\{\alpha_{1}, \ldots, \alpha_{l}\right\}$. Let $(\alpha \mid \beta)$ denote the inner product on $\Phi$ normalized such that $(\alpha \mid \alpha)=2$ for a long root. We set $\alpha^{\vee}:=\frac{2 \alpha}{(\alpha \mid \alpha)}$ and $\langle\alpha, \beta\rangle:=\left(\alpha \mid \beta^{\vee}\right)$. We extend $\langle$,$\rangle to a pairing between the root$ lattice and the co-root lattice $\Lambda$.

Let $\mathfrak{g}$ be the corresponding simple Lie algebra over $\mathbb{Q}$. Fix a Chevalley basis in g. It defines a simply connected group Chevalley group $\underline{G}$. It is an algebraic group defined over $\mathbb{Z}$. For any field $F$ the group $G(F)$ is generated by one-parameter subgroups $\underline{U}_{\alpha} \simeq F$ for every root $\alpha$ in $\Phi$. More precisely, the choice of Chevalley basis fixes an isomorphism of $F$ and $\underline{U}_{\alpha}, t \mapsto \underline{e}_{\alpha}(t)$ for every $t \in F$. For example, if $G=\mathrm{SL}_{2}$, then $\underline{e}_{\alpha}(t)$ and $\underline{e}_{-\alpha}(t)$ are

$$
\left(\begin{array}{ll}
1 & t \\
0 & 1
\end{array}\right) \text { and }\left(\begin{array}{cc}
1 & 0 \\
t & 1
\end{array}\right)
$$


respectively. Define elements

$$
\left\{\begin{array}{l}
\underline{w}_{\alpha}(t)=\underline{e}_{\alpha}(t) \underline{e}_{-\alpha}\left(-t^{-1}\right) \underline{e}_{\alpha}(t), \\
\underline{h}_{\alpha}(t)=\underline{w}_{\alpha}(t) \underline{w}_{\alpha}(-1) .
\end{array}\right.
$$

If $G=\mathrm{SL}_{2}$, then these elements are

$$
\left(\begin{array}{cc}
0 & t \\
-t^{-1} & 0
\end{array}\right) \text { and }\left(\begin{array}{cc}
t & 0 \\
0 & t^{-1}
\end{array}\right)
$$

By a result of Steinberg (Theorem 8(b), page 66 in $[\mathrm{St}$ ), the group $\underline{G}(F)$ is abstractly generated by the one-parameter groups $\underline{U}_{\alpha}$ modulo relations

$$
\left[\underline{e}_{\alpha}(t), \underline{e}_{\beta}(u)\right]= \begin{cases}\prod_{i, j \geq 1} \underline{e}_{i \alpha+j \beta}\left(c_{i j} t^{i} u^{j}\right) & \text { if } \alpha+\beta \text { is a root } \\ 1 & \text { if not, and }-\alpha \neq \beta\end{cases}
$$

and

$$
\underline{h}_{\alpha}(s) \underline{h}_{\alpha}(t)=\underline{h}_{\alpha}(s t),
$$

where $c_{i j}$ are integers depending on $\alpha, \beta$.

Now assume that $F=\mathbb{R}$ or $\mathbb{Q}_{p}$. Let $(\cdot, \cdot)$ be the Hilbert symbol 11 over $F$. It defines a two-fold central extension $G(F)$,

$$
1 \rightarrow \mu_{2} \rightarrow G(F) \stackrel{\operatorname{pr}}{\rightarrow} \underline{G}(F) \rightarrow 1,
$$

by replacing the relation (2) by

$$
h_{\alpha}(s) h_{\alpha}(t)=h_{\alpha}(s t) \cdot(s, t)^{\frac{1}{2}\left(\alpha^{\vee} \mid \alpha^{\vee}\right)} .
$$

Indeed, Steinberg (Theorem 12, page 86 in $[\mathrm{St}$ ) shows that a two-fold central extension of $\underline{G}(F)$ is necessarily defined by these generators and relations, while Matsumoto $\mathrm{Ma}$ proves existence of the central extension.

Let $U_{\alpha}$ be the subgroup of $G(F)$ generated by $e_{\alpha}(t)$. Then $U_{\alpha} \simeq \underline{U}_{\alpha}$ and the splitting is unique since $F$ is two-divisible. Important to us will be the subgroups $G_{\alpha}$ generated by $U_{\alpha}$ and $U_{-\alpha}$. Let $\underline{G}_{\alpha} \cong \mathrm{SL}_{2}$ be the projection of $G_{\alpha}$ in $\underline{G}$. Since $\left[h_{\alpha}(t), e_{\alpha}(u)\right]=e_{\alpha}\left(\left(t^{2}-1\right) u\right)$, the group $G_{\alpha}$ is perfect. Thus $G_{\alpha}$ is a central extension of $\underline{G}_{\alpha}$ of degree $m_{\alpha}$. It follows from (3) that $m_{\alpha}=2$ except when $\alpha$ is a short root in $\mathrm{B}_{n}, \mathrm{C}_{n}$ or $\mathrm{F}_{4}$; then $m_{\alpha}=1$. Indeed, if $\alpha$ is a short root in $\mathrm{B}_{n}, \mathrm{C}_{n}$ or $\mathrm{F}_{4}$, then $\left(\alpha^{\vee} \mid \alpha^{\vee}\right)=4$ and there is no Hilbert symbol in (3).

The group $G\left(\mathbb{Z}_{p}\right)$ is a (preferred) hyperspecial maximal compact subgroup of $\underline{G}\left(\mathbb{Q}_{p}\right)$. It stabilizes the Chevalley lattice and is generated by $e_{\alpha}(t)$ with $t$ in $\mathbb{Z}_{p}$. By reducing modulo $p$ we have an exact sequence

$$
1 \rightarrow K_{p}^{1} \rightarrow \underline{G}\left(\mathbb{Z}_{p}\right) \rightarrow \underline{G}\left(\mathbb{F}_{p}\right) \rightarrow 1
$$

Proposition 2.1. The central extension splits over $\underline{G}\left(\mathbb{Z}_{p}\right)$ for $p \neq 2$. The splitting homomorphism $s: \underline{G}\left(\mathbb{Z}_{p}\right) \rightarrow G\left(\mathbb{Q}_{p}\right)$ is unique and its image is henceforth denoted by $K_{p}$.

\footnotetext{
${ }^{1}$ For reference: The Hilbert symbol over $\mathbb{Q}_{2}$ is given by $\left(2^{\alpha} u, 2^{\beta} v\right)_{2}=(-1)^{r}$, where $u, v \in 1+2 \mathbb{Z}_{2}$ and $r=\left(\frac{u-1}{2}\right)\left(\frac{v-1}{2}\right)+\alpha \frac{v^{2}-1}{8}+\beta \frac{u^{2}-1}{8}$. The symbol over $\mathbb{Q}_{p}$ is $\left(p^{\alpha} u, p^{\beta} v\right)_{p}=$ $(-1)^{r}\left(\frac{u}{p}\right)^{\beta}\left(\frac{v}{p}\right)^{\alpha}$, where $u, v \in \mathbb{Z}_{p}^{\times}$and $r=\alpha \beta\left(\frac{p-1}{2}\right)$.
} 
Proof. As the proof of Lemma 11.3 in $\mathrm{Mo}$ shows, the central extension splits over the pro- $p$ subgroup $K_{p}^{1}$. Hence the central extension of $\underline{G}\left(\mathbb{Q}_{p}\right)$ gives rise to a central extension of the finite group $\underline{G}\left(\mathbb{F}_{p}\right)$. However, the group $\underline{G}\left(\mathbb{F}_{p}\right)$ has no Schur multipliers of order 2 if $p$ is odd and the group is not of type $\mathrm{B}_{3} \mathrm{Gr}$. This proves that the central extension splits over the hyperspecial maximal compact subgroup except perhaps for the type $\mathrm{B}_{3}$. However, a splitting for the type $\mathrm{B}_{4}$ implies a splitting for the type $\mathrm{B}_{3}$, by inclusion of the corresponding groups.

It remains to show that the splitting is unique. Any two splittings differ by a homomorphism from $\underline{G}\left(\mathbb{Z}_{p}\right)$ to $\mu_{2}$. Such a homomorphism is clearly trivial on the pro- $p$ group $K_{p}^{1}$, and then it must be trivial on $\underline{G}\left(\mathbb{F}_{p}\right)$ since it has no normal subgroups of index $Z$. (Both arguments rely on the fact that $p \neq 2$.)

Proposition 2.2. If $p$ is odd, then $K_{p}$ contains $e_{\alpha}(t)$ for all $t \in \mathbb{Z}_{p}$ and, therefore, $h_{\alpha}(t)$ for all $t \in \mathbb{Z}_{p}^{\times}$.

Proof. Note that $U_{\alpha}$ and $K_{p}$ give two splittings of $\underline{U}_{\alpha}\left(\mathbb{Z}_{p}\right)$. They differ by a quadratic character of $\mathbb{Z}_{p}$. Since $\mathbb{Z}_{p}$ is 2-divisible if $p \neq 2$, the character is trivial.

Let $S$ be any finite set of places containing $\{\infty, 2\}$. Let

$$
\mu_{S}=\left\{\left(\epsilon_{1}, \ldots, \epsilon_{|S|}\right) \in \mu_{2}^{|S|}: \epsilon_{1} \cdots \epsilon_{|S|}=1\right\} .
$$

Define

$$
G_{S}=\left(\prod_{v \in S} G\left(\mathbb{Q}_{v}\right)\right) / \mu_{S} \times \prod_{v \notin S} K_{v} .
$$

If $S \subseteq S^{\prime}$, then $G_{S} \subseteq G_{S^{\prime}}$. We define $G(\mathbb{A})$ as a direct limit of all $G_{S}$. We have a central extension

$$
1 \rightarrow \mu_{2} \rightarrow G(\mathbb{A}) \rightarrow \underline{G}(\mathbb{A}) \rightarrow 1
$$

For every $\alpha \in \Phi$ and $t \in \mathbb{Q}, e_{\alpha}(t)$ can be viewed as an element in $G(\mathbb{A})$ by diagonal embedding. This is well-defined by Proposition 2.2. These elements clearly satisfy the relations in (11). Moreover, corresponding $h_{\alpha}(t)$ 's satisfy the relations in (2) by quadratic reciprocity for the Hilbert symbol. In particular, we have an explicit splitting of the extension over $\underline{G}(\mathbb{Q})$.

Maximal compact $K_{\infty}$. There is an automorphism $\sigma$ of $G(\mathbb{R})$ such that $\sigma$ : $e_{\alpha}(t) \mapsto e_{-\alpha}(-t)$ for every root $\alpha$ and $t \in \mathbb{R}$ (see Thm. 16 in [St]). The fixed points of $\sigma$ on $\underline{G}(\mathbb{R})$ is a maximal compact subgroup $K_{\infty}$. Similarly there is an automorphism $\underline{\sigma}$ of $\underline{G}(\mathbb{R})$, and its fixed points $\underline{K}_{\infty}$ are a maximal compact subgroup of $\underline{G}(\mathbb{R})$.

\section{The torus}

Let $\underline{T} \subseteq G$ be the maximal split torus. If $R$ is a ring, then $\underline{T}(R)$ is generated by $\underline{h}_{\alpha}(t)$ with $t \in R^{\times}$. If $\Lambda$ is the co-root lattice, then $\underline{T}(R) \simeq \Lambda \otimes_{\mathbb{Z}} R^{\times}$with the isomorphism given by

$$
\underline{h}_{\alpha}(t) \mapsto \alpha^{\vee} \otimes t
$$

Let $T(F) \subset G(F)$ be the inverse image of $\underline{T}(F)$. Then $T(F)$ is generated by $h_{\alpha}(t)$. The following commutator formula ([Ma, Lemme 5.4) is crucial to us throughout the paper:

$$
\left[h_{\alpha}(s), h_{\beta}(t)\right]=(s, t)^{\left(\alpha^{\vee} \mid \beta^{\vee}\right)} .
$$


The goal of this section is to describe the structure of $T(F)$ for $F=\mathbb{R}$ and $F=\mathbb{Q}_{p}$ and to define pseudo-spherical representations of $T(\mathbb{R})$ and $T\left(\mathbb{Q}_{2}\right)$, and unramified representations of $T\left(\mathbb{Q}_{p}\right)$ for $p$ odd.

Case $F=\mathbb{Q}_{p}$, with $p$ odd. Define $T_{p}=T\left(\mathbb{Q}_{p}\right) \cap K_{p}$. Then by Proposition 2.2. $T_{p}$ is generated by $h_{\alpha}(t)$ for all $t \in \mathbb{Z}_{p}^{\times}$and is isomorphic to $\underline{T}\left(\mathbb{Z}_{p}\right)$ by $h_{\alpha}(t) \mapsto \underline{h}_{\alpha}(t)$. Note that the symbol $(\cdot, \cdot)$ is tame here, i.e. $h_{\alpha}(s) h_{\alpha}(t)=h_{\alpha}(s t)$ for all $s, t \in \mathbb{Z}_{p}^{\times}$. Let $T_{p}^{2}$ be the set of squares in $T_{p}$. Critical to us are the genuine representations of $T\left(\mathbb{Q}_{p}\right)$ which are trivial on $T_{p}^{2}$. A genuine representation of $T\left(\mathbb{Q}_{p}\right)$ is unramified if it has a non-zero vector fixed by $T_{p}$.

Case $F=\mathbb{R}$. We note that $(-1,-1)=-1$. In this case $\underline{T}(\mathbb{R})=M \underline{A}$, where $\underline{M} \simeq \Lambda \otimes\{ \pm 1\}$ and $\underline{A} \simeq \Lambda \otimes \mathbb{R}^{+}$. Then $T(\mathbb{R})=M A$, where $M$ is generated by $h_{\alpha}(-1)$ and contains the kernel $\mu_{2}$ of the central extension. On the other hand $A$ is generated by $h_{\alpha}(t)$ for $t \in \mathbb{R}^{+}$and $A \simeq \underline{A}$. Note also that $A$ is in the center of $T(\mathbb{R})$. Thus it is natural to concentrate on genuine representations of $M$. Let $M_{s}$ be the subgroup of $M$ generated by $h_{\alpha}(-1)$ for all roots $\alpha$ such that $m_{\alpha}=1$. Since $h_{\alpha}(-1) h_{\alpha}(-1)=1$ for such roots, $M_{s}$ does not contain the central subgroup $\mu_{2} \subset M$. An irreducible genuine representation of $M$ trivial on the normal subgroup $M_{s}$ is called a pseudo-spherical representation. An important feature of pseudospherical representations of $M$ is that they are invariant under the conjugation action of the Weyl group. See Lemma 4.11(3) in $[\mathrm{A}-\mathrm{V}$.

Case $F=\mathbb{Q}_{2}$. This is the most interesting case. The Hilbert symbol is ramified. The group $\mathbb{Z}_{2}^{\times}$has a filtration

$$
\mathbb{Z}_{2}^{\times}=1+2 \mathbb{Z}_{2} \supseteq 1+4 \mathbb{Z}_{2} \supseteq 1+8 \mathbb{Z}_{2} .
$$

Note that $1+8 \mathbb{Z}_{2}=\left(\mathbb{Z}_{2}^{\times}\right)^{2}$. In particular, $1+8 \mathbb{Z}_{2}$ is in the kernel of the Hilbert symbol. Since $\mathbb{Z}_{2}^{\times} /\left(1+8 \mathbb{Z}_{2}\right) \simeq(\mathbb{Z} / 8 \mathbb{Z})^{\times}=\{ \pm 1, \pm 5\}$, all values of the symbol are easily obtained from the following table.

\begin{tabular}{|r||r|r|r|}
\hline & 2 & -1 & 5 \\
\hline \hline 2 & 1 & 1 & -1 \\
\hline-1 & 1 & -1 & 1 \\
\hline 5 & -1 & 1 & 1 \\
\hline
\end{tabular}

Observe that the kernel of the symbol $(\cdot, \cdot)$ when restricted to $\mathbb{Z}_{2}^{\times}$is $1+4 \mathbb{Z}_{2}$. For every integer $i \geq 1$, let $\underline{T}_{2}^{i}$ be the subgroup of $\underline{T}\left(\mathbb{Z}_{2}\right)$ isomorphic to

$$
\underline{T}_{2}^{i} \simeq \Lambda \otimes\left(1+2^{1+i} \mathbb{Z}_{2}\right) .
$$

Let $T\left(\mathbb{Z}_{2}\right) \subset G\left(\mathbb{Q}_{2}\right)$ be the inverse image of $\underline{T}\left(\mathbb{Z}_{2}\right)$. Since the Hilbert symbol is trivial on $1+4 \mathbb{Z}_{2}$, for every $i \geq 1$, elements $h_{\alpha}(t)$ for $t \in 1+2^{1+i} \mathbb{Z}_{2}$ generate a subgroup $T_{2}^{i} \subset T\left(\mathbb{Z}_{2}\right)$ isomorphic to $\underline{T}_{2}^{i}$. Note that $T_{2}^{1}$ is contained in the center of $T\left(\mathbb{Z}_{2}\right)$, while $T_{2}^{2}$ is contained in the center of $T\left(\mathbb{Q}_{2}\right)$.

Since $(-1,-1)_{2}=(-1,-1)_{\infty}=-1$, the subgroup of $T\left(\mathbb{Z}_{2}\right)$ generated by $h_{\alpha}(-1)$ is isomorphic to $M$ of the real case! Moreover, since the non-trivial coset of $1+4 \mathbb{Z}_{2}$ in $1+2 \mathbb{Z}_{2}=\mathbb{Z}_{2}^{\times}$is represented by -1 , we have an isomorphism

$$
T\left(\mathbb{Z}_{2}\right) \simeq M \times T_{2}^{1} .
$$

As in the real case, let $M_{s}$ be the subgroup of $M$ generated by $h_{\alpha}(-1)$ for all roots $\alpha$ such that $m_{\alpha}=1$. Then $M_{s} T_{2}^{1}$ is a commutative subgroup of $T\left(\mathbb{Q}_{2}\right)$. Note that 
this group is generated by $h_{\alpha}(t)$, where $t \in 1+4 \mathbb{Z}_{2}$ if $\alpha$ is long and $t \in \mathbb{Z}_{2}^{\times}$if $\alpha$ is short. We say that a genuine representation of $T\left(\mathbb{Q}_{2}\right)$ is pseudo-spherical if it has a vector invariant under $M_{s} T_{2}^{1}$.

Weyl groups. Assume that $F=\mathbb{R}$ or $\mathbb{Q}_{p}$. Let $W_{F}$ denote the subgroup of $G(F)$ generated by $w_{\alpha}(1)$ for all simple roots $\alpha$. Let $T_{F}(\mathbb{Z})$ denote the subgroup generated by $h_{\alpha}(-1)$ for all simple roots $\alpha$. Let $W$ denote the Weyl group of $G(\mathbb{Q})$. Then we have an exact sequence

$$
1 \rightarrow T_{F}(\mathbb{Z}) \rightarrow W_{F} \rightarrow W \rightarrow 1 .
$$

Conjugation action of $W_{F}$ on $T(F)$ does not descend to that of $W$ because $T_{F}(\mathbb{Z})$ does not lie in the center of $T(F)$. Suppose $(\pi, V)$ is a representation of $T(F)$ and $w \in W_{F}$. Let $V^{w}$ denote the representation defined by $t \mapsto \pi\left(w^{-1} t w\right)$. Note that the isomorphism class of $V^{w}$ depends only on the projection of $w$ into the Weyl group $W$. In other words, we have a conjugation action of the Weyl group on the set of isomorphism classes of irreducible representations of $T(F)$. The following lemma implies that the classes of pseudo-spherical and unramified representations are preserved under the conjugation action of the Weyl group.

Proposition 3.1. The following subgroups of $T(F)$ are normalized by $W_{F}$ :

(i) $T_{p}$ if $F=\mathbb{Q}_{p}$ and $p$ is an odd prime.

(ii) $T_{2}^{1}$ and $M_{s}$ if $F=\mathbb{Q}_{2}$.

(iii) $A$ and $M_{s}$ if $F=\mathbb{R}$.

Proof. Combining (3) and Lemma 37(c) in St] gives

$$
w_{\alpha}(1) h_{\beta}(t) w_{\alpha}(-1)=h_{\kappa}(t) \cdot(c, t)^{\frac{1}{2}\left(\beta^{\vee} \mid \beta^{\vee}\right)}
$$

where $\kappa=w_{\alpha}(\beta)$ and $c= \pm 1$, which depends on structure coefficients for the Chevalley basis. In order to prove the proposition we need to show that the sign after $h_{\kappa}(t)$ is trivial for $h_{\alpha}(t)$ generating the relevant groups. If $h_{\alpha}(t)$ is in $T_{p}, T_{2}^{1}$ or $A$, then $(c, t)=1$ by elementary properties of the Hilbert symbol. Finally, recall that $M_{s}$ is generated by $h_{\beta}(-1)$, where $\beta$ is a root such that $\left(\beta^{\vee} \mid \beta^{\vee}\right)=4$. Thus the sign is trivial in here, too.

\section{Representations of $T(F)$}

Assume that $H$ is a subgroup of $G$ which is the inverse image of an abelian subgroup $\underline{H}$ in $\underline{G}$. Assume furthermore that the center $Z(H)$ of $H$ has finite index in $H$. Let $\mathbf{H}=H / Z(H)$ and $q: H \rightarrow \mathbf{H}$ denote the quotient map. Since $\underline{H}$ is abelian, the square of any element of $H$ is contained in $\mu_{2} \subseteq Z(H)$. It follows that $\mathbf{H} \simeq(\mathbb{Z} / 2 \mathbb{Z})^{r}$, and we may consider $\mathbf{H}$ as a vector space over $\mathbb{Z} / 2 \mathbb{Z}$. Given $\mathbf{x}=q(x), \mathbf{y}=q(y) \in \mathbf{H}$ for some $x, y \in H$, we define $B(\mathbf{x}, \mathbf{y})=x y x^{-1} y^{-1} \in \mu_{2}$. The definition of $B$ is independent of the choice of $x, y$, and $B$ could be interpreted as a symplectic non-degenerate form on $\mathbf{H}$. In particular, we may write $\mathbf{H}=\mathbf{H}_{1} \oplus \mathbf{H}_{2}$ as a direct sum of isotropic subspaces with respect to $B$, and $\operatorname{dim} \mathbf{H}=r$ is even. We define $H_{1}=q^{-1} \mathbf{H}_{1}$, which is an abelian subgroup of $H$ containing $Z(H)$.

Recall that an irreducible representation of $H$ (resp. $Z(H)$ ) is called genuine if it is non-trivial on the kernel $\mu_{2}$ of the covering map. Let $\operatorname{Irr}_{\text {gen }}(H)$ be the set of equivalence classes of irreducible genuine finite dimensional representations of $H$, and let $\operatorname{Irr}_{\text {gen }}(Z(H))$ be the set of genuine characters of $Z(H)$. 
Proposition 4.1. Given $H$ and $Z(H)$ as above. Then there is a one-to-one correspondence between $\operatorname{Irr}_{\text {gen }}(H)$ and $\operatorname{Irr}_{\text {gen }}(Z(H))$ given by sending an irreducible genuine representation of $H$ to its central character. Moreover, the dimension of every genuine irreducible representation is equal to the square root of the index of $Z(H)$ in $H$.

Proof. This is essentially Proposition 2.2 in $\mathrm{A}-\mathrm{V}$. Let $V \in \operatorname{Irr}_{\text {gen }}(H)$. Let $\chi_{V}$ denote its character, which is well defined since $V$ is finite dimensional. The exact same argument as in $\mathrm{A}-\mathrm{V}$ shows that $\chi_{V}$ is supported on $Z(H)$. By Proposition 3 in Chapter 8, Section 12 in Bou, the isomorphism class of $V$ is uniquely determined by $\chi_{V}$. Hence the isomorphism class of $V$ is uniquely determined by its central character in $\operatorname{Irr}_{\text {gen }}(Z(H))$.

Conversely, given $\chi \in \operatorname{Irr}_{\text {gen }}(Z(H))$, we can extend $\chi$ to a one dimensional character $\tilde{\chi}$ of $H_{1}$. Indeed we may choose $\tilde{\chi}$ to be an irreducible $H_{1}$-submodule of $\operatorname{Ind}_{Z(H)}^{H_{1}} \chi$. By Mackey theory, $\operatorname{Ind}_{H_{1}}^{H} \tilde{\chi}$ is an irreducible representation of $H$ of dimension $[H: Z(H)]^{1 / 2}$ with central character $\chi$.

We apply this proposition to the group $M$, which is the inverse image of $\underline{M}$. In order to describe the center $Z(M)$ of $M$ we need to consider the commutator map on $M$, which induces a (symmetric) $\mu_{2}$-valued pairing on $\underline{M} \cong \Lambda \otimes\{ \pm 1\} \cong \Lambda / 2 \Lambda$. Since the commutator is given by

$$
\left[h_{\alpha}(-1), h_{\beta}(-1)\right]=(-1,-1)_{2}^{\left(\alpha^{\vee} \mid \beta^{\vee}\right)},
$$

the pairing is (the same as) the bilinear form $(\cdot \mid \cdot)$ reduced modulo 2 . The kernel is given by the lattice $\Lambda \cap 2 \Lambda^{*}$, where $\Lambda^{*} \supseteq \Lambda$ is the dual lattice with respect to the form $(\cdot \mid \cdot)$. In particular, the index of $\mu_{2}$ in $Z(M)$ is equal to the index $\left[\Lambda \cap 2 \Lambda^{*}: 2 \Lambda\right]$, and the index of $Z(M)$ in $M$ is equal to the index $\left[\Lambda: \Lambda \cap 2 \Lambda^{*}\right]$. By Proposition 4.1 we have proved the following:

Proposition 4.2. The number of irreducible genuine representations of $M$ is equal to the index $\left[\Lambda \cap 2 \Lambda^{*}: 2 \Lambda\right]$. The dimension of each such representation is a square root of the index of $\left[\Lambda: \Lambda \cap 2 \Lambda^{*}\right]$.

In the following table we give the index of $\Lambda \cap 2 \Lambda^{*}$ in $\Lambda$ in the simply laced case and $\mathrm{G}_{2}$ :

\begin{tabular}{|c||c|c|c|c|c|c|c|c|}
\hline$\Phi$ & $\mathrm{A}_{2 n-1}$ & $\mathrm{~A}_{2 n}$ & $\mathrm{D}_{2 n-1}$ & $\mathrm{D}_{2 n}$ & $\mathrm{E}_{6}$ & $\mathrm{E}_{7}$ & $\mathrm{E}_{8}$ & $\mathrm{G}_{2}$ \\
\hline$\left[\Lambda: \Lambda \cap 2 \Lambda^{*}\right]$ & $4^{n-1}$ & $4^{n}$ & $4^{n-1}$ & $4^{n-1}$ & $4^{3}$ & $4^{3}$ & $4^{4}$ & 4 \\
\hline
\end{tabular}

The index for types $\mathrm{B}_{l}, \mathrm{C}_{l}$ and $\mathrm{F}_{4}$ is the same as the index for $\mathrm{A}_{l-1}, \mathrm{~A}_{1}$ and $\mathrm{A}_{2}$, respectively. In other words, it is the same as the index for the subsystem generated by simple long roots.

In order to discuss genuine irreducible representations of $T\left(\mathbb{Q}_{p}\right)$, we need to describe the center of $T\left(\mathbb{Q}_{p}\right)$. We fix a choice of simple roots $\triangle=\left\{\alpha_{1}, \ldots, \alpha_{l}\right\}$. If $\lambda=n_{1} \alpha_{1}^{\vee}+\cdots+n_{l} \alpha_{l}^{\vee}$ is an element in the co-root lattice $\Lambda$, then we define

$$
\eta(\lambda):=h_{\alpha_{1}}\left(p^{n_{1}}\right) \cdots h_{\alpha_{l}}\left(p^{n_{l}}\right) \in T\left(\mathbb{Q}_{p}\right) .
$$

We shall use $\eta_{p}$ instead of $\eta$ if there is a need to distinguish between primes. Note that the order of multiplication is important, as the $h_{\alpha_{i}}\left(p^{n_{i}}\right)$ 's may not commute with one another. Indeed, the commutator is given by

$$
\left[\eta(\lambda), \eta\left(\lambda^{\prime}\right)\right]=(p, p)^{\left(\lambda \mid \lambda^{\prime}\right)},
$$


which may be non-trivial since $(p, p)=-1$ if $p \equiv 3(\bmod 4)$. If $\Lambda^{\prime}$ is a subset of $\Lambda$, then we set $\eta\left(\Lambda^{\prime}\right):=\left\{\eta(\lambda): \lambda \in \Lambda^{\prime}\right\}$.

Case $p$ is odd. Note that we have a decomposition $T\left(\mathbb{Q}_{p}\right)=T_{p} \cdot \eta(\Lambda) \cdot \mu_{2}$. The commutator of $h_{\alpha}(p)$ in $\eta(\Lambda)$ and $h_{\beta}(t)$ in $T_{p}$ is

$$
\left[h_{\alpha}(p), h_{\beta}(t)\right]=(p, t)_{p}^{\left(\alpha^{\vee} \mid \beta^{\vee}\right)} .
$$

Since $(p, t)_{p}=1$ if and only if $t$ is a square in $\mathbb{Z}_{p}^{\times}$, it follows that the commutator defines a pairing of $\Lambda \times T_{p} / T_{p}^{2} \cong \Lambda \times \Lambda / 2 \Lambda$ which is simply the restriction of the bilinear form $(\cdot \mid \cdot)$ modulo 2 . This shows that the centralizer of $T_{p}$ in $\eta(\Lambda)$ is $\eta\left(\Lambda \cap 2 \Lambda^{*}\right)$ and the centralizer of $\eta(\Lambda)$ in $T_{p}$ is the group $C_{p}$ containing $T_{p}^{2}$, and such that $C_{p} / T_{p}^{2} \cong\left(\Lambda \cap 2 \Lambda^{*}\right) / 2 \Lambda$. It follows that the center of $T\left(\mathbb{Q}_{p}\right)$ is $Z_{p}=C_{p} \cdot \eta\left(\Lambda \cap 2 \Lambda^{*}\right) \cdot \mu_{2}$. Note that the index of $Z_{p}$ in $T\left(\mathbb{Q}_{p}\right)$ is $\left[\Lambda: \Lambda \cap 2 \Lambda^{*}\right]^{2}$. The next proposition follows from Proposition 4.1 .

Proposition 4.3. There is a bijection between genuine irreducible representations $V$ of $T\left(\mathbb{Q}_{p}\right)$ and genuine characters $\gamma$ of $Z_{p}$, the center of $T\left(\mathbb{Q}_{p}\right)$. Moreover, any such representation $V$ has the dimension equal to the index $\left[\Lambda: \Lambda \cap 2 \Lambda^{*}\right]$.

If $\gamma$ is a genuine character of $Z_{p}$, the corresponding representation of $T\left(\mathbb{Q}_{p}\right)$ will be henceforth denoted by $V(\gamma)$. Let $\operatorname{Irr}_{\text {gen }}^{2}\left(T\left(\mathbb{Q}_{p}\right)\right)$ be the set of isomorphism classes of genuine representations of $T\left(\mathbb{Q}_{p}\right)$ with non-zero $T_{p}^{2}$-fixed vectors. Define an equivalence relation on $\operatorname{Irr}_{\text {gen }}^{2}\left(T\left(\mathbb{Q}_{p}\right)\right)$, where two representations $V$ and $V^{\prime}$ are equivalent if $V^{\prime}$ is isomorphic to a twist of $V$ by an unramified character of the algebraic torus $\underline{T}\left(\mathbb{Q}_{p}\right)$.

Proposition 4.4. Two genuine representations $V(\gamma)$ and $V\left(\gamma^{\prime}\right)$ in $\operatorname{Irr}_{\text {gen }}^{2}\left(T\left(\mathbb{Q}_{p}\right)\right)$ are equivalent if and only if $\left.\gamma\right|_{C_{p}}=\left.\gamma^{\prime}\right|_{C_{p}}$. The number of equivalence classes is equal to the index $\left[\Lambda \cap 2 \Lambda^{*}: 2 \Lambda\right]$. Only one of these classes, the class where $\left.\gamma\right|_{C_{p}}=1$, consists of unramified representations of $T\left(\mathbb{Q}_{p}\right)$.

Proof. Since $Z_{p}=C_{p} \cdot \eta\left(\Lambda \cap 2 \Lambda^{*}\right) \cdot \mu_{2}$, it easily follows that any two genuine characters of $Z_{p}$ which coincide on $C_{p}$ are unramified twists of one another. It follows that the equivalence classes are parameterized by characters of the finite group $C_{p} / T_{p}^{2}$. Since the order of this group is $\left[\Lambda \cap 2 \Lambda^{*}: 2 \Lambda\right]$, we have proved the first two statements. If $V(\gamma)$ is unramified, that is, it contains a vector fixed by $T_{p}$, then the central character must be trivial on $C_{p}$. The proposition is proved.

Case $p=2$. The set $T^{1}\left(\mathbb{Q}_{2}\right):=T_{2}^{1} \cdot \eta_{2}(\Lambda) \cdot \mu_{2}$ is a normal subgroup of $T\left(\mathbb{Q}_{2}\right)$ and commutes with $M$, as can be seen from the values of the Hilbert symbol $(\cdot, \cdot)_{2}$. Thus

$$
T\left(\mathbb{Q}_{2}\right)=\left(M \times T^{1}\left(\mathbb{Q}_{2}\right)\right) / \mu_{2} .
$$

It follows that any genuine representation of $T\left(\mathbb{Q}_{2}\right)$ is a tensor product of genuine representations of $M$ and $T^{1}\left(\mathbb{Q}_{2}\right)$. Moreover, we have the following key proposition which reduces the study of representations of $T\left(\mathbb{Q}_{2}\right)$ to that of $M$ and $T\left(\mathbb{Q}_{p}\right)$ for $p \equiv 1(\bmod 4)$.

Proposition 4.5. Assume that $p \equiv 1(\bmod 4)$. Pick a non-square $\zeta$ in $\mathbb{F}_{p}^{\times}$. The map given by $h_{\alpha}(2) \mapsto h_{\alpha}(p)$ and $h_{\alpha}(5) \mapsto h_{\alpha}(\zeta)$ induces an isomorphism

$$
T^{1}\left(\mathbb{Q}_{2}\right) / T_{2}^{2} \cong T\left(\mathbb{Q}_{p}\right) / T_{p}^{2} .
$$


Proof. This is obvious since the tame symbol $(\cdot, \cdot)_{p}$ takes the following values:

\begin{tabular}{|r||r|r|}
\hline & $p$ & $\zeta$ \\
\hline \hline$p$ & 1 & -1 \\
\hline$\zeta$ & -1 & 1 \\
\hline
\end{tabular}

\section{Modular forms on $T(\mathbb{A})$}

We are interested in studying Eisenstein series on $G(\mathbb{A})$. To that end we need to understand the space $\mathcal{A}=L_{\text {gen }}^{2}(A \underline{T}(\mathbb{Q}) \backslash T(\mathbb{A}))$. It is natural to look for maximally unramified representations in $\mathcal{A}$ first. Recall that $T_{p}=K_{p} \cap T\left(\mathbb{Q}_{p}\right)$ if $p$ is odd and $T_{2}^{1}$ is generated by $h_{\alpha}(t)$ for all simple roots $\alpha$ and $t \in 1+4 \mathbb{Z}_{2}$.

Proposition 5.1. Let $\mathcal{A}_{0}$ be the space of all right $T_{2}^{1} \prod_{p \neq 2} T_{p}$-invariant functions in $\mathcal{A}$. Note that this is naturally an $M \times M$ module where the two factors sit in $T(\mathbb{R})$ and $T\left(\mathbb{Z}_{2}\right)$. As such it is isomorphic to the genuine part of the regular representation of the finite group $M$ :

$$
\mathcal{A}_{0} \cong L_{\text {gen }}^{2}(M) \text {. }
$$

Proof. In the proof, $h_{\alpha, \mathbb{Q}}(t), h_{\alpha, \infty}(t)$ and $h_{\alpha, p}(t)$ denote elements of the global group $\underline{T}(\mathbb{Q})$ and the local groups $T(\mathbb{R})$ and $T\left(\mathbb{Q}_{p}\right)$, respectively. Let $I$ be the group of invertible adeles. In view of the decomposition

$$
I=\mathbb{Q}^{\times} \cdot \mathbb{R}^{+} \times \prod_{p} \mathbb{Z}_{p}^{\times}
$$

the space $\mathcal{A}_{0}$ is indeed isomorphic to $L_{\text {gen }}^{2}(M)$, where here $M$ is considered as a subgroup of $T\left(\mathbb{Z}_{2}\right)$. In order to finish the proof we need to determine the action of $h_{\alpha, \infty}(-1)$ for this identification. Let $f$ be in $\mathcal{A}_{0}$. Since $f$ is left $\underline{T}(\mathbb{Q})$ and right $T_{p}$-invariant, for every $p \neq 2$, for every $m$ in $T\left(\mathbb{Z}_{2}\right)$ we have

$$
f\left(m h_{\alpha, \infty}(-1)\right)=f\left(h_{\alpha, \mathbb{Q}}(-1)^{-1} m h_{\alpha, \infty}(-1)\right)=f\left(h_{\alpha, 2}(-1)^{-1} m\right) .
$$

Recall that $M_{s} \subseteq M$ is generated by $h_{\alpha, 2}(-1)$ for all roots $\alpha$ such that $m_{\alpha}=1$. In particular it is a central subgroup. Now let $\mathcal{A}_{00}$ be the subspace of $\mathcal{A}_{0}$ consisting of $M_{s}$-invariant functions. Let $\bar{M}=M / M_{s}$ be the quotient group. By the PeterWeyl theorem, we have

$$
\mathcal{A}_{00} \cong L_{\text {gen }}^{2}(\bar{M})=\bigoplus_{\delta} \delta \otimes \delta^{\vee},
$$

where the sum is taken over irreducible genuine representations $\delta$ of $\bar{M}$ or, equivalently, over the pseudo-spherical representations of $M$. Thus we have the following corollary:

Corollary 5.2. Let $\delta$ be a pseudo-spherical representation of $M$. Then there exists a unique representation $\pi \subseteq L_{\text {gen }}^{2}(A \underline{T}(\mathbb{Q}) \backslash T(\mathbb{A}))$ such that $\pi_{\infty} \cong \delta$ and $\pi_{p}$ is unramified at all primes. The isomorphism class of $\pi_{p}$ is invariant under the conjugation of the Weyl group. 
Proof. The uniqueness is obvious. Now consider a Weyl group conjugate $\pi^{w}$. Note that $\pi^{w}$ is again unramified at all primes. Since $\delta^{w} \cong \delta$ it follows that $\pi^{w} \cong \pi$ by the uniqueness of $\pi$.

Let $\pi$ be the global representation as in the previous corollary. We would like to determine the local components $\pi_{p}$. To that end we need to determine the corresponding central characters. A large part of the center acts trivially on $\pi$, independent of the choice of $\delta$ :

Proposition 5.3. Let $p$ be any prime. For any $t$ in $\mathbb{Q}_{p}^{\times}$the central element $h_{\alpha, p}\left(t^{m_{\alpha}}\right)$ acts trivially on $\mathcal{A}_{00}$.

Proof. Since $\mathcal{A}_{00}$ is $\left(M_{s} T_{2}^{1}\right) \prod_{p \neq 2} T_{p}$-right invariant, it suffices to check this for $t=p$. Assume first that $p$ is odd. Let $f$ be in $\mathcal{A}_{00}$. Note that $f$ is right $h_{\alpha, q}\left(p^{m_{\alpha}}\right)$ invariant for every $q \neq p$. Indeed, $h_{\alpha, q}\left(p^{m_{\alpha}}\right)$ is contained in $T_{q}$ if $q \neq 2$ and in $M_{s} T_{2}^{1}$ if $q=2$. (This is clear if $m_{\alpha}=1$; otherwise it follows from $p^{2} \equiv 1(\bmod 4)$ for every odd $p$.) Using left $h_{\alpha, \mathbb{Q}}\left(p^{m_{\alpha}}\right)$-invariance of $f$ we have

$$
f\left(m h_{\alpha, p}\left(p^{m_{\alpha}}\right)\right)=f\left(h_{\alpha, \mathbb{Q}}\left(p^{m_{\alpha}}\right)^{-1} m h_{\alpha, p}\left(p^{m_{\alpha}}\right)\right)=f(m) .
$$

Now assume that $p=2$. Then, analogously,

$$
f\left(m h_{\alpha, 2}\left(2^{m_{\alpha}}\right)\right)=f\left(h_{\alpha, \mathbb{Q}}\left(2^{m_{\alpha}}\right)^{-1} m h_{\alpha, 2}\left(2^{m_{\alpha}}\right)\right)=f(m) .
$$

In order to determine the central character of $\pi_{p}$, we need to determine the action of the full center of $T\left(\mathbb{Q}_{p}\right)$ on $\delta \otimes \delta^{\vee} \subseteq \mathcal{A}_{00}$. Observe that $(p, p)_{p}=(p, p)_{2}=$ $(-1)^{(p-1) / 2}$ for any odd prime. This allows us to define a homomorphism

$$
\varphi: \eta_{p}(\Lambda) \cdot \mu_{2} \rightarrow T\left(\mathbb{Z}_{2}\right)
$$

by sending $h_{\alpha, p}(p)$ to $h_{\alpha, 2}(p)$. The restriction of $\varphi$ to $\eta_{p}\left(\Lambda \cap 2 \Lambda^{*}\right)$ has the image in the center of $T\left(\mathbb{Z}_{2}\right)$. Thus, if $\gamma_{\infty}$ is the central character of $\delta$, then the composite

$$
\gamma_{p}=\gamma_{\infty} \circ \varphi
$$

defines an unramified central character for $T\left(\mathbb{Q}_{p}\right)$. We also define $\gamma_{2}$, an unramified central character of $T^{1}\left(\mathbb{Q}_{2}\right)$, by $\gamma_{2}\left(\eta_{2}(\lambda)\right)=1$ for any $\lambda$ in $\Lambda \cap 2 \Lambda^{*}$.

Proposition 5.4. Fix a pseudo-spherical representation $\delta$ of $M$. Let $\pi \subseteq \mathcal{A}$ be the unique representation such that $\pi_{\infty} \cong \delta$ and that $\pi_{p}$ is unramified for all primes $p$, as in Corollary [5.2. Let $\gamma_{p}$ be the central character defined by (5). Then $\pi_{2} \cong$ $\delta^{\vee} \otimes V\left(\gamma_{2}\right)$ and $\pi_{p} \cong V\left(\gamma_{p}\right)$ for $p$ odd.

Proof. The proof is completely analogous to the proof of Proposition 5.3 . We leave the details to the reader.

For uniformity, we set $\gamma_{\infty}$ to be the central character of $\pi_{\infty}=\delta$ extended trivially to $A$. We set $V\left(\gamma_{\infty}\right)$ to be the representation $\delta$ extended trivially to $A$. 


\section{Principal series Representations of $G\left(\mathbb{Q}_{v}\right)$}

In this section we define principal series representations of $G\left(\mathbb{Q}_{v}\right)$, where $v=\infty$ or $p$. Let $B=T U$ denote the Borel subgroup of $G$, where $U$ is generated by $e_{\alpha}(t)$ for all positive roots $\alpha$. Let $\bar{U}$ be the group generated by $e_{\alpha}(t)$ for all negative roots $\alpha$.

Fix a pseudo-spherical representation $\delta$ of $M$. It gives rise to a global representation $\pi$ of $T(\mathbb{A})$, such that $\pi_{\infty} \cong \delta$ as in Corollary [5.2. Let $\chi$ be an unramified character of $\underline{T}\left(\mathbb{Q}_{v}\right)$. If $v=\infty$ an unramified character is a character trivial on $\underline{M}$. Let $i(\chi)$ be the twist of $\pi_{v}$ by $\chi$. Since $\pi_{v}$ is Weyl group invariant, we have $i(\chi)^{w} \cong i\left(\chi^{w}\right)$ for every $w$ in $W$. In this section we study induced representations (normalized induction)

$$
I(\chi)=\operatorname{Ind}_{B}^{G}(i(\chi))
$$

Let $\alpha$ be a simple root. A character $\chi$ is called $\alpha$-dominant if $\chi\left(\underline{h}_{\alpha}(t)\right)=|t|^{s}$ with $\Re(s)>0$. A character $\chi$ is called dominant if it is $\alpha$-dominant for all simple roots. For every $w$ in $W_{\mathbb{Q}_{v}}$ we have an intertwining map $A_{w}: I(\chi) \rightarrow I\left(\chi^{w}\right)$ defined by

$$
A_{w}(f)(g)=\int_{U \cap w \bar{U} w^{-1}} f\left(w^{-1} u g\right) d u .
$$

Proposition 6.1. The operator $A_{w}$ is absolutely convergent if $\chi$ is dominant.

Proof. Our proof is, of course, based on the corresponding result for algebraic groups. (See, for example, Section 2.1 of $[\mathrm{Sh}$.$) Let \ell(w)$ denote the length of the projection of $w$ into the Weyl group. The proof of the proposition is on induction on the length $\ell(w)$. We consider the case of $\ell(w)=1$. Then $w$ corresponds to a simple root, so we shall denote it by $w_{\alpha}$.

Lemma 6.2. Let $\alpha$ be a simple root and $\chi$ an unramified $\alpha$-dominant character of $\underline{T}$. Then $A_{w_{\alpha}}$ is absolutely convergent.

Proof. The proof of this lemma is a reduction to $\mathrm{SL}_{2}$. Let $s \in \mathbb{C}$ such that $\chi\left(\underline{h}_{\alpha}(t)\right)=|t|^{s}$. Then $\Re(s)>0$ since $\chi$ is $\alpha$ dominant. In the formula for $A_{w_{\alpha}}(f)$ we can assume that $g=1$, replacing $f$ if necessary. Note that $U \cap w_{\alpha} \bar{U} w_{\alpha}^{-1}=U_{\alpha}$; thus the question of convergence is answered by working in $G_{\alpha}$. Let $B_{\alpha}=B \cap G_{\alpha}=$ $T_{\alpha} U_{\alpha}$. The restriction of $f$ to $G_{\alpha}$ belongs to the induced representation $\operatorname{Ind}_{B_{\alpha}}^{G_{\alpha}}(i(\chi))$. Note that $T_{\alpha}$, the group generated by elements $h_{\alpha}(t)$, is commutative. Decompose $i(\chi)=\bigoplus \mu_{i}$ as a sum of characters of $T_{\alpha}$. It follows that $\operatorname{Ind}_{B_{\alpha}}^{G_{\alpha}}(i(\chi))=\bigoplus I_{i}$, where $I_{i}$ are principal series representations induced from the characters $\mu_{i}$. Recall that $i(\chi)=\pi_{p} \otimes \chi$. Since Proposition 5.3 describes the action of $h_{\alpha}(t)$ on $\pi_{p}$, it follows that

$$
\left|\mu_{i}\left(h_{\alpha}(t)\right)\right|=|t|^{\Re(s)}
$$

for every $i$ and $\alpha$. Thus, if we write $f=\bigoplus f_{i}$ with $f_{i}$ in $I_{i}(s)$, then $\left|f_{i}\right|$ belongs to a principal series representation $I(\Re(s))$ of $\underline{G}_{\alpha} \cong \mathrm{SL}_{2}$, induced from the character $\underline{h}_{\alpha}(t) \mapsto|t|^{\Re(s)}$. The convergence of the integral for $\left|f_{i}\right|$ can be easily calculated. If $\mathbb{Q}_{v}=\mathbb{R}$ the integral is bounded by a multiple of

$$
\int_{\mathbb{R}}\left(\frac{1}{1+x^{2}}\right)^{\frac{\Re(s)+1}{2}} d x
$$


while if $v=p$, then the integral is bounded by a multiple of

$$
\sum_{i=n}^{\infty} \frac{1}{p^{n \Re(s)}}
$$

Both of these converge if $\Re(s)>0$.

Now we can easily finish the proof of the proposition. Assume that $\chi$ is dominant and $A_{w}$ is absolutely convergent for some $w$ in $W$. If $\ell\left(w_{\alpha} w\right)=\ell(w)+1$, then $\chi^{w}$ is $\alpha$-dominant. In particular, the composite $A_{w_{\alpha}} \circ A_{w}$ is absolutely convergent. It is equal to $A_{w_{\alpha} w}$ by Fubini's theorem. The proposition is proved.

Recall that $m_{\alpha}$ is the degree of the central extension $G_{\alpha}$ of $\underline{G}_{\alpha} \cong \mathrm{SL}_{2}$. This number is equal to 2 , except when $\alpha$ is the short root in the root systems $\mathrm{C}_{n}, \mathrm{~B}_{n}$ and $\mathrm{F}_{4}$. A character $\chi_{0}: \underline{T}\left(\mathbb{Q}_{v}\right) \rightarrow \mathbb{R}^{+}$such that $\chi_{0}\left(\underline{h}_{\alpha}(t)\right)=|t|^{\frac{1}{m_{\alpha}}}$ for every simple root $\alpha$ is called exceptional. Note that $\chi_{0}$ is unique and dominant.

Proposition 6.3. The induced representation $I\left(\chi_{0}\right)$ has a unique quotient. We denote the quotient by $\Theta\left(\gamma_{v}\right)$.

Proof. When $v$ is the archimedean place, $\Theta\left(\gamma_{\infty}\right)$ is the Langlands quotient of $I\left(\chi_{0}\right)$.

Suppose $v=p$. In this case this is a standard result for induced representations with a regular inducing character. More precisely, we say that $i(\chi)$ is regular if $i(\chi)$ is not isomorphic to $i\left(\chi^{w}\right)$ for any non-trivial element $w$ in the Weyl group. If that is the case, then $I(\chi)$ has a unique irreducible submodule and, dually, a unique irreducible quotient. This can be seen as follows. By the geometric lemma in [BZ, the semi-simplification of the (unnormalized) Jacquet module $I(\chi)_{U}$ is

$$
I(\chi)_{U} \cong \bigoplus_{w \in W}\left[\rho_{U} \cdot i\left(\chi^{w}\right)\right],
$$

where $\rho_{U}$ is the square root of the modular character with respect to $U$. Suppose $V$ is an irreducible submodule of $I(\chi)$. Then, by Frobenius reciprocity, $\operatorname{Hom}_{G}(V, I(\chi))=\operatorname{Hom}_{T}\left(V_{U}, \rho_{U} \cdot i(\chi)\right)$, so $\rho_{U} \cdot i(\chi)$ must be a summand of $V_{U}$. By exactness of the Jacquet functor and regularity of $i(\chi), V$ must be unique. This proves the proposition.

Remark. For $G\left(\mathbb{Q}_{v}\right)$ of type $\mathrm{C}_{n}$, the exceptional representation $\Theta\left(\gamma_{v}\right)$ is an even component of the oscillator representation [W]. The representation $\pi_{v}=V\left(\gamma_{v}\right)=$ $\gamma_{v}$ is one dimensional and is the Weil index [Rao.

If $v=p$, then the Jacquet functor $\Theta\left(\gamma_{p}\right)_{U}$ can be exactly described.

Proposition 6.4. Let $\chi_{0}$ be the exceptional character and $w_{0}$ the longest element in the Weyl group. Then $\Theta\left(\gamma_{p}\right)_{U} \cong \rho_{U} \cdot i\left(\chi_{0}^{w_{0}}\right)$.

Proof. Let $\alpha$ be a simple root. Let $P_{\alpha}=G_{\alpha} \cdot B$ be a parabolic subgroup, where $G_{\alpha}$ is the group generated by one-parameter subgroups $U_{\alpha}$ and $U_{-\alpha}$. We need the following lemma:

Lemma 6.5. For every simple root $\alpha$, the induced representation $\operatorname{Ind}_{B}^{P_{\alpha}}\left(i\left(\chi_{0}\right)\right)$ is reducible.

Proof. Let us restrict this representation to $G_{\alpha}$. Decompose $i(\chi)=\bigoplus \mu_{i}$ as a sum of characters of $T_{\alpha}=G_{\alpha} \cap T$. It follows that $\operatorname{Ind}_{B}^{P_{\alpha}}\left(i\left(\chi_{0}\right)\right)=\bigoplus I_{i}$, where $I_{i}$ are principal series representations of $G_{\alpha}$, parabolically induced from the characters 
$\mu_{i}$. The characters $\mu_{i}$ are determined as follows. Recall that $i\left(\chi_{0}\right)$ is a twist, by $\chi_{0}$, of a Weyl-group invariant representation of $T\left(\mathbb{Q}_{p}\right)$ appearing as a local component of a representation in $\mathcal{A}$. Hence, if $m_{\alpha}=1$, then Proposition 5.3 implies that $\mu_{i}\left(h_{\alpha}(t)\right)=\chi_{0}\left(h_{\alpha}(t)\right)=|t|$. It follows that each $I_{i}$ has the Steinberg representation as a submodule and the trivial representation as a quotient. Since $T$ normalizes $G_{\alpha}$, the sum of all Steinberg submodules is a proper submodule for $P_{\alpha}$. A similar argument works if $m_{\alpha}=2$. Then Proposition 5.3 implies that $\mu_{i}\left(h_{\alpha}\left(t^{2}\right)\right)=\chi_{0}\left(h_{\alpha}\left(t^{2}\right)\right)=|t|$. It follows that each $I_{i}$ reduces with a discrete series representation as a submodule and a quotient isomorphic to an even component of an oscillator representation [GS]. Again, the sum of discrete series representations is a $P_{\alpha}$-submodule. The lemma is proved.

We now follow an argument of Rodier Ro. Let $V_{\alpha}$ be the unique quotient of $\operatorname{Ind}_{B}^{P_{\alpha}}\left(i\left(\chi_{0}\right)\right)$. Then, by induction in stages, $\operatorname{Ind}_{P_{\alpha}}^{G}\left(V_{\alpha}\right)$ is a quotient of $I\left(\chi_{0}\right)$. Since $\Theta\left(\gamma_{p}\right)$ is the unique irreducible quotient of $I\left(\chi_{0}\right)$, it must also be a quotient of $\operatorname{Ind}_{P_{\alpha}}^{G}\left(V_{\alpha}\right)$. Since

$$
\operatorname{Ind}_{P_{\alpha}}^{G}\left(V_{\alpha}\right)_{U}=\bigoplus_{w \in W, w(\alpha)<0}\left[\rho_{U} \cdot i\left(\chi_{0}^{w}\right)\right]
$$

it follows that $\Theta\left(\gamma_{p}\right)_{U}$ is a sum of $\rho_{U} \cdot i\left(\chi_{0}^{w}\right)$ for $w$ in the Weyl group such that $w(\alpha)$ is negative for all simple roots $\alpha$. But this holds only for $w=w_{0}$, the longest element in the Weyl group. The proposition is proved.

Assume that $p$ is odd. Let $v^{\circ}$ be a non-zero element in $i(\chi)$ fixed by $T_{p}$. Note that $v^{\circ}$ is unique up to a non-zero scalar. Then the representation $I(\chi)$ contains a unique $K_{p}$-fixed vector $f_{\chi}^{\circ}$ normalized by $f_{\chi}^{\circ}(1)=v^{\circ}$. The action of the intertwining operators on the spherical vector has been computed in Sa2].

Proposition 6.6. Assume that $p \neq 2$. Let $\alpha$ be a simple root. Then

$$
A_{w_{\alpha}}\left(f_{\chi}^{\circ}\right)=\frac{1-p^{-1}\left(\chi\left(h_{\alpha}\left(p^{m_{\alpha}}\right)\right)\right)}{1-\chi\left(h_{\alpha}\left(p^{m_{\alpha}}\right)\right)} f_{\chi^{w_{\alpha}}}^{\circ} .
$$

Note that the formula for $A_{w_{\alpha}(1)}\left(f_{\chi}^{\circ}\right)$ depends on the projection of $w_{\alpha}$ into the Weyl group $W$. Thus, for a general element in $W_{\mathbb{Q}_{p}}$ we have the following corollary.

Corollary 6.7. Let $\underline{w}$ be in $W$ and $w$ be a preimage of $\underline{w}$ in $W_{\mathbb{Q}_{p}}$. Then

$$
A_{w}\left(f_{\chi}^{\circ}\right)=\prod_{\alpha>0, \underline{w}(\alpha)<0} \frac{1-p^{-1}\left(\chi\left(h_{\alpha}\left(p^{m_{\alpha}}\right)\right)\right)}{1-\chi\left(h_{\alpha}\left(p^{m_{\alpha}}\right)\right)} f_{\chi^{w}}^{\circ} .
$$

\section{EISENSTEIN SERIES}

Recall that $B=T U$ denotes the Borel subgroup of $G$, where $U$ is generated by $e_{\alpha}(t)$ for all positive root $\alpha$. In the same fashion, we define the Borel subgroup $\underline{B}=\underline{T U}$ of $\underline{G}$.

We identify $\mathbb{A}^{l} \simeq \underline{T}(\mathbb{A})$ by $\left(x_{1}, \ldots, x_{l}\right) \mapsto \prod_{i=1}^{l} h_{\alpha_{i}}\left(x_{i}\right)$. For $\mathbf{s}=\left(s_{1}, \ldots, s_{l}\right) \in \mathbb{C}^{l}$, we define the Hecke character $\chi_{\mathbf{s}}$ of $\underline{T}(\mathbb{Q}) \backslash \underline{T}(\mathbb{A})$ by $\chi_{\mathbf{s}}\left(\underline{h}_{\alpha_{i}}\left(x_{i}\right)\right)=\left|x_{i}\right|^{s_{i}}$ for every

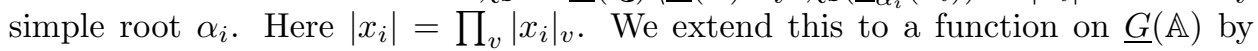
$\chi_{\mathbf{s}}(u t k)=\chi_{\mathbf{s}}(t)$, where $u \in \underline{U}(\mathbb{A}), t \in \underline{T}(\mathbb{A})$ and $k \in \prod_{p} K_{\infty} \underline{G}\left(\mathbb{Z}_{p}\right)$. The square root of the modular function is given by $\rho=\chi_{(1, \ldots, 1)}=\chi_{\mathbf{1}}$, where $\mathbf{1}=(1, \ldots, 1)$. 
Similarly, for a place $v$ of $\mathbb{Q}$, we define a character $\chi_{\mathbf{s}, v}$ of $\underline{T}\left(\mathbb{Q}_{v}\right)$ by $\chi_{\mathbf{s}, v}\left(\underline{h}_{\alpha_{i}}(t)\right)=$ $|t|_{v}^{s_{i}}$ for every simple root $\alpha_{i}$. We extend this to a function on $\underline{G}\left(\mathbb{Q}_{v}\right)$ by $\chi_{\mathbf{s}, v}(u t k)=$ $\chi_{\mathbf{s}, v}(t)$, where $u \in \underline{U}\left(\mathbb{Q}_{v}\right), t \in \underline{T}\left(\mathbb{Q}_{v}\right)$ and $k \in \underline{K}_{v}$.

Let $\pi$ be as in Corollary [5.2. Let $K=K_{\infty} \prod_{p} K_{p}$. Let $\mathcal{J}$ denote the space of functions on $G(\mathbb{A})$ satisfying the following conditions:

(1) $f(u b a g)=f(g)$ for $u \in U(\mathbb{A}), b \in B(\mathbb{Q}), a \in A, g \in G(\mathbb{A})$,

(2) $f$ is $K$-finite, and for each $k \in K$, the function $t \mapsto f(t k)$ is a function in $\pi$. Let $I\left(\chi_{\mathbf{s}}\right)$ denote the representation of $G(\mathbb{A})$ on functions of the form

$$
g \mapsto f(g) \chi_{\mathbf{s}+\mathbf{1}}(g),
$$

where $f \in \mathcal{J}$. We have

$$
I\left(\chi_{\mathbf{s}}\right)=\operatorname{Ind}_{B(\mathbb{A})}^{G(\mathbb{A})} \pi \chi_{\mathbf{s}}=\left(\operatorname{Ind}_{B(\mathbb{R})}^{G(\mathbb{R})} \pi_{\infty} \chi_{\mathbf{s}, \infty}\right) \bigotimes_{p} \operatorname{Ind}_{B\left(\mathbb{Q}_{p}\right)}^{G\left(\mathbb{Q}_{p}\right)} \pi_{p} \chi_{\mathbf{s}, p},
$$

where all the induced representations are normalized inductions. We form an Eisenstein series:

$$
E(g, \mathbf{s}, f)=\sum_{x \in \underline{B}(\mathbb{Q}) \backslash \underline{G}(\mathbb{Q})} f(x g) \chi_{\mathbf{s}+\mathbf{1}}(g),
$$

where $g \in G(\mathbb{A}), \mathbf{s} \in \mathbb{C}^{l}, f \in \mathcal{J}$. The above sum converges absolutely and uniformly on compact sets contained in the region $\operatorname{Re}\left(s_{i}\right)>1$ for all $i$. The Eisenstein series has a meromorphic continuation to $\mathbb{C}^{l}$; see [MW. We define the constant term of the above Eisenstein series by

$$
E(g, \mathbf{s}, f)_{U}=\int_{U(\mathbb{Q}) \backslash U(\mathbb{A})} E(u g, \mathbf{s}, f) d u .
$$

A standard computation in the domain of convergence of $E(g, \mathbf{s}, f)$ gives

$$
E(g, \mathbf{s}, f)_{U}=\sum_{\underline{w} \in W}\left(A_{w}(\mathbf{s}) f\right)(g),
$$

where

$$
\left(A_{w}(\mathbf{s}) f\right)(g)=\int_{\left(U(\mathbb{Q}) \cap w \bar{U}(\mathbb{Q}) w^{-1}\right) \backslash\left(U(\mathbb{A}) \cap w \bar{U}(\mathbb{A}) w^{-1}\right)} f\left(w^{-1} u g\right) \chi_{\mathbf{s}+\mathbf{1}}\left(w^{-1} u g\right) d u
$$

and $w \in W_{\mathbb{Q}}$ is an (arbitrary) element such that $\operatorname{pr}(w)=\underline{w}$. Suppose $S$ is a finite set of primes including 2 and $\infty$ and $f=\left(\bigotimes_{v \in S} f_{v}\right) \otimes\left(\bigotimes_{p \notin S} f_{p}^{\circ}\right)$. Then by Corollary 6.7

$$
\left(A_{w}(\mathbf{s}) f\right)(g)=\left(\bigotimes_{v \in S} A_{w, v}(\mathbf{s}) f_{v}\right) \otimes\left(c_{S}(\underline{w}, \mathbf{s}) \bigotimes_{p \notin S} f_{w(\mathbf{s}), p}^{\circ}\right)
$$

where

$$
c_{S}(\underline{w}, \mathbf{s})=\prod_{p \notin S} \prod_{\alpha>0, \underline{w}(\alpha)<0} \frac{1-p^{-1}\left(\chi_{\mathbf{s}, p}\left(h_{\alpha}\left(p^{m_{\alpha}}\right)\right)\right)}{1-\chi_{\mathbf{s}, p}\left(h_{\alpha}\left(p^{m_{\alpha}}\right)\right)}=\prod_{\alpha>0, \underline{w}(\alpha)<0} \frac{\zeta_{S}\left(m_{\alpha} \alpha(\mathbf{s})\right)}{\zeta_{S}\left(1+m_{\alpha} \alpha(\mathbf{s})\right)} .
$$

Here $\zeta_{S}(z)=\prod_{p \notin S}\left(1-p^{-s}\right)^{-1}$ is the partial Riemann zeta function, and $\alpha(\mathbf{s})=$ $\sum_{i=1}^{l} n_{i} s_{i}$ if $\alpha=\sum_{i=1}^{l} n_{i} \alpha_{i}$ as a sum of simple roots. Therefore as $\mathbf{s}$ tends to $\mathbf{s}_{0}=\left(m_{\alpha_{1}}^{-1}, \ldots, m_{\alpha_{l}}^{-1}\right)$, each term $\left(\prod_{i=1}^{l}\left(s_{i}-m_{\alpha_{i}}^{-1}\right)\right) A_{w}(\mathbf{s}) f$ vanishes except for the term where $\underline{w}=\underline{w}_{0}$ is the longest element of $W$. Furthermore, if we set $S=\{2, \infty\}$, then $A_{w, v}\left(\mathbf{s}_{0}\right)$ for $v \in S$ are non-zero intertwining operators, so we may arrange $f$ such that $\left(\prod_{i=1}^{l}\left(s_{i}-m_{\alpha_{i}}^{-1}\right)\right) A_{w}(\mathbf{s}) f$ is non-zero. 
For $f \in \mathcal{J}$, we define

$$
\theta_{f}(g)=\lim _{\mathbf{s} \rightarrow \mathbf{s}_{0}}\left(\prod_{i=1}^{l}\left(s_{i}-m_{\alpha_{i}}^{-1}\right)\right) E(g, \mathbf{s}, f) .
$$

Then

$$
\int_{U(\mathbb{Q}) \backslash U(\mathbb{A})} \theta_{f}(u g) d u=A_{w_{0}}\left(\mathbf{s}_{0}\right)(f),
$$

and, by the criterion of Jacquet (see [J] and [MW]), $\theta_{f}(g)$ is a square integrable function in $L^{2}(\underline{G}(\mathbb{Q}) \backslash G(\mathbb{A}))$. Let $\Theta$ denote the span of $\left\{\theta_{f}: f \in \mathcal{J}\right\}$. We now recall the exceptional representation $\Theta\left(\gamma_{v}\right)$ defined in Section 6 .

Theorem 7.1. The span $\Theta$ lies in $L^{2}(\underline{G}(\mathbb{Q}) \backslash G(\mathbb{A}))$. It is an irreducible automorphic representation of $G(\mathbb{A})$, and it is isomorphic to $\bigotimes_{v} \Theta\left(\gamma_{v}\right)$.

Proof. For every $f \in \mathcal{J}$, the map $f \chi_{\mathbf{s}_{0}+\mathbf{1}} \mapsto \theta_{f}$ defines a non-zero intertwining operator from the induced representation to $L^{2}(\underline{G}(\mathbb{Q}) \backslash G(\mathbb{A}))$. Thus the image $\Theta$ must decompose as a direct sum of irreducible representations. On the other hand, at each local place $v$ the exceptional representation $\Theta\left(\gamma_{v}\right)$ is a unique quotient of the local induced representation. This implies that $\Theta \cong \bigotimes_{v} \Theta\left(\gamma_{v}\right)$, as desired.

Corollary 7.2. The exceptional representation $\Theta\left(\gamma_{v}\right)$ is unitarizable.

In a terminology of $[\mathrm{A}-\mathrm{V}], \Theta\left(\gamma_{\infty}\right)$ corresponds to the trivial representation of a split group $\underline{G}^{l}(\mathbb{R})$, which will be introduced in the next section. The unitarity of $\Theta\left(\gamma_{\infty}\right)$ was proved and studied for classical groups of type $\mathrm{B}_{l}$ in $[\mathrm{Kn},[\mathrm{LS}]$ and $[\mathrm{T}]$. The unitarity for other groups may be new.

\section{IWAHORI-HECKE ALGEBRAS}

We will fix an odd prime $p$ in this section. We fix an Iwahori subgroup $I$ of $K_{p}$ such that $I$ contains $U_{\alpha}\left(\mathbb{Z}_{p}\right)$ for all positive $\alpha$ and $I \cap T\left(\mathbb{Q}_{p}\right)=T_{p}$. We recall that $\mu_{2}$ is the kernel of the covering map pr: $G\left(\mathbb{Q}_{p}\right) \rightarrow \underline{G}\left(\mathbb{Q}_{p}\right)$. Let $\mathcal{H}_{-}=\mathcal{H}_{-}\left(G\left(\mathbb{Q}_{p}\right)\right)$ denote the algebra of all compactly supported $I$-bi-invariant functions on $G\left(\mathbb{Q}_{p}\right)$ such that $f(\epsilon g)=\epsilon f(g)$ for all $\epsilon \in \mu_{2}$. The multiplicative structure of $\mathcal{H}_{-}$is defined by a convolution of functions,

$$
\left(f^{\prime} \cdot f^{\prime \prime}\right)(g)=\int_{G} f^{\prime}(h) f^{\prime \prime}\left(h^{-1} g\right) d h,
$$

where $d h$ is a Haar measure on $G$ so that the volume of $\mu_{2} \times I$ is one. We call $\mathcal{H}_{-}$ the Iwahori-Hecke algebra of $G$. The following is Proposition 6.1 in Sa2.

Proposition 8.1. Let $N^{\prime}$ denote the normalizer in $G$ of $T_{p}$. Then the support of the Hecke algebra is $\operatorname{supp}\left(\mathcal{H}_{-}\right)=I N^{\prime} I$.

One can easily describe $N^{\prime}$. Recall that, if $\underline{N}\left(\mathbb{Q}_{p}\right)$ is the normalizer of $\underline{T}\left(\mathbb{Z}_{p}\right)$ in $G\left(\mathbb{Q}_{p}\right)$, then the quotient of the two is isomorphic to the affine Weyl group $\Lambda \rtimes W$. The group $N^{\prime}$ is smaller than the inverse image of $\underline{N}\left(\mathbb{Q}_{p}\right)$. Recall that $\eta_{p}(\lambda)$ centralizes (or normalizes) $T_{p}$ if and only if $\lambda$ is in

$$
\Lambda^{\prime}:=\Lambda \cap 2 \Lambda^{*} .
$$

In particular, we have an exact sequence

$$
1 \rightarrow \mu_{2} \times T_{p} \rightarrow N^{\prime} \stackrel{\phi}{\rightarrow} \Lambda^{\prime} \rtimes W \rightarrow 1,
$$


where $\phi$ is defined by sending $w_{\alpha}(1)$ to the reflection $w_{\alpha}$ in $W$ and $\eta_{p}(\lambda)$ to $\lambda$ in $\Lambda^{\prime}$.

We now define a normalization of elements in the Hecke algebra. Let $\pi_{p}$ be an unramified, Weyl group invariant, irreducible genuine representation of $T\left(\mathbb{Q}_{p}\right)$ as in Corollary 5.2. Let $\gamma_{p}$ be the central character of $\pi_{p}$. Recall that $\eta_{p}(\lambda)$ is in the center of $T\left(\mathbb{Q}_{p}\right)$ for every $\lambda$ in $\Lambda^{\prime}$. In particular, $\gamma_{p}\left(\eta_{p}(\lambda)\right)$ is well defined for every $\lambda$ in $\Lambda^{\prime}$. The Weyl group invariance of the central character of $\pi_{p}$ implies that we can extend $\gamma_{p}$ to $N^{\prime}$ by setting

$$
\gamma_{p}\left(w_{\alpha}(1)\right)=1 \text {. }
$$

Thus, $\gamma_{p}$ is a character of $N^{\prime}$, which is trivial on $T_{p}$. For $w$ in $\Lambda^{\prime} \rtimes W$, we define $e_{w} \in \mathcal{H}_{-}$by it values for every $x$ in $N^{\prime}$ as follows:

$$
e_{w}(I x I)=\left\{\begin{array}{l}
\overline{\gamma_{p}(x)} \text { if } \phi(x)=w, \\
0 \text { otherwise }
\end{array}\right.
$$

We note some elementary properties of elements $e_{w}$. Let $\ell(w)$ denote the usual length function on the affine Weyl group $\Lambda \rtimes W$. If $\ell\left(w_{1} w_{2}\right)=\ell\left(w_{1}\right)+\ell\left(w_{2}\right)$ for two elements in $\Lambda^{\prime} \rtimes W$, then $e_{w_{1} w_{2}}=e_{w_{1}} \cdot e_{w_{2}}$. (See [Sa2]. A key here is the multiplicative property of $\gamma_{p}$.)

Let $\mathcal{L}$ denote the $\mathbb{C}$-span of $e_{\lambda}$ where $\lambda$ is dominant in $\Lambda^{\prime}$. Note that $\ell(\lambda)=\langle\rho, \lambda\rangle$ for dominant $\lambda$. It follows that $\ell\left(\lambda+\lambda^{\prime}\right)=\ell(\lambda)+\ell\left(\lambda^{\prime}\right)$ for dominant $\lambda, \lambda^{\prime}$ in $\Lambda^{\prime}$. Hence $e_{\lambda} \cdot e_{\lambda^{\prime}}=e_{\lambda+\lambda^{\prime}}$. In particular, $\mathcal{L}$ is a commutative subalgebra in $\mathcal{H}_{-}$.

Let $H$ denote the subalgebra consisting of functions supported on $\mu_{2} \times K_{p}$. It has basis $\left\{e_{w}: w \in W\right\}$. If $\alpha$ is a simple root and $w_{\alpha}$ is the corresponding simple reflection, then we denote $e_{w_{\alpha}}$ by $e_{\alpha}$. These elements satisfy the following relations:

(1) $\left(e_{\alpha}-p\right)\left(e_{\alpha}+1\right)=0$ and

(2) $e_{\alpha} \cdot e_{\beta} \cdot e_{\alpha} \cdots=e_{\beta} \cdot e_{\alpha} \cdot e_{\beta} \cdots$, where the number of factors on each side is equal to the order $m_{\alpha \beta}$ of the element $w_{\alpha} w_{\beta}$ in $W$.

Conversely, $H$ is the $\mathbb{C}$-algebra generated by the set of $e_{\alpha}$ for all simple roots $\alpha$ satisfying the above two relations. One easily sees that

$$
\mathcal{H}_{-}=H \cdot \mathcal{L} \cdot H \text {. }
$$

An important result is that for a positive $\lambda \in \Lambda^{\prime}, e_{\lambda}$ is an invertible element in $\mathcal{H}_{-}$. This implies that if $V$ is an admissible genuine $G$-module generated by the subspace $V^{I}$, then every submodule $V_{1}$ of $V$ is also generated by its subspace $V_{1}^{I}$.

Given $\lambda \in \Lambda^{\prime}$, we write $\lambda=\lambda_{1}-\lambda_{2}$, where $\lambda_{1}, \lambda_{2}$ are positive in $\Lambda^{\prime}$. We define

$$
t_{\lambda}=p^{-\frac{1}{2}\langle\rho, \lambda\rangle} e_{\lambda_{1}} \cdot e_{\lambda_{2}}^{-1} \text {. }
$$

This definition does not depend on the choice of $\lambda_{1}$ and $\lambda_{2}$. We state the main results of [Sa1] and [Sa2]. (Note that we have already explained the first three relations.)

Theorem 8.2. Let $\alpha, \beta$ be two simple roots, and let $\lambda, \lambda^{\prime} \in \Lambda^{\prime}$. Then $e_{\alpha}, e_{\beta}, t_{\lambda}$ and $t_{\lambda^{\prime}}$ satisfy the following relations:

(1) $\left(e_{\alpha}-p\right)\left(e_{\alpha}+1\right)=0$.

(2) $e_{\alpha} \cdot e_{\beta} \cdot e_{\alpha} \cdots=e_{\beta} \cdot e_{\alpha} \cdot e_{\beta} \cdots$, where there are $m_{\alpha \beta}$ factors on each side.

(3) $t_{\lambda} \cdot t_{\lambda^{\prime}}=t_{\lambda+\lambda^{\prime}}$.

(4) $e_{\alpha} \cdot t_{\lambda}-t_{w_{\alpha}(\lambda)} \cdot e_{\alpha}=(q-1) \frac{t_{\lambda}-t_{w_{\alpha}(\lambda)}}{1-t_{-m_{\alpha} \alpha^{\vee}}}$. 
Conversely, let $\mathcal{H}_{-}^{\prime}$ be the $\mathbb{C}$-algebra abstractly generated by $e_{\alpha}$ for all simple roots $\alpha$, and $t_{\lambda}$ for all $\lambda \in \Lambda^{\prime}$. These generators satisfy the relations (1) to (4) above; then $\mathcal{H}_{-}^{\prime}=\mathcal{H}_{-}$.

Remark. The above theorem was stated in $\underline{\mathrm{Sa} 2}$ for simply laced $\underline{G}$, and for any degree central extension. However, the proof of relation (4) takes place in the Levi factor of the parabolic subgroup $P_{\alpha}$. Thus the calculation given there (relying on $\gamma_{p}\left(h_{\alpha}\left(p^{m_{\alpha}}\right)\right)=1$; Proposition 5.3) is applicable to our situation.

Definition of $\underline{G}^{l}$. We will define an algebraic split group $\underline{G}^{l}\left(\mathbb{Q}_{p}\right)$. In order to do this, it suffices to define its co-roots $\Psi^{\vee}$ and its co-character lattice $\Lambda_{c}$. We recall that $\Lambda$ is the co-root lattice of $G$, and we define

$$
\Psi^{\vee}:=\left\{\frac{m_{\alpha}}{2} \alpha^{\vee} \in \Lambda \otimes \mathbb{R} \mid \alpha^{\vee} \in \Phi^{\vee}\right\}
$$

and $\Lambda_{c}:=\frac{1}{2} \Lambda^{\prime}$. Note that the root system $\Psi$ is dual to the root system $\Phi$. The isogeny class of $\underline{G}^{l}$ is determined by the lattice $\Lambda_{c}$. Let $\Lambda_{c r}$ be the $\mathbb{Z}$-span of coroots in $\Psi^{\vee}$. The group $\underline{G}^{l}$ is a split, algebraic group obtained by taking a quotient of the split, simply connected algebraic group corresponding to $\Psi$ by the central subgroup isomorphic to $\Lambda_{c} / \Lambda_{c r}$. It is an elementary 2-group. Its order is equal to the number of pseudo-spherical representations of $M$. The following table lists all cases when this 2-group is non-trivial:

\begin{tabular}{|c||c|c|c|c|c|c|}
\hline$\Phi$ & $\mathrm{A}_{2 n-1}$ & $\mathrm{D}_{2 n-1}$ & $\mathrm{D}_{2 n}$ & $\mathrm{C}_{n}$ & $\mathrm{~B}_{2 n}$ & $\mathrm{E}_{7}$ \\
\hline$\Psi$ & $\mathrm{A}_{2 n-1}$ & $\mathrm{D}_{2 n-1}$ & $\mathrm{D}_{2 n}$ & $\mathrm{~B}_{n}$ & $\mathrm{C}_{2 n}$ & $\mathrm{E}_{7}$ \\
\hline$\left[\Lambda_{c}: \Lambda_{c r}\right]$ & 2 & 2 & 4 & 2 & 2 & 2 \\
\hline
\end{tabular}

The Iwahori-Hecke algebra $\mathcal{H}\left(\underline{G}^{l}\right)$ of $\underline{G}^{l}$ is similarly generated by $\underline{t}_{\lambda}$ and $\underline{e}_{w}$, where $\lambda \in \Lambda_{c}$ and $w \in W$.

Let $f(x) \in \mathcal{H}(G)\left(\right.$ resp. $\left.\mathcal{H}\left(\underline{G}^{l}\right)\right)$. We define $f^{*}(x)=\overline{f\left(x^{-1}\right)}$. Hence $*: \mathcal{H}_{-} \rightarrow \mathcal{H}_{-}$ (resp. * : $\mathcal{H}\left(\underline{G}^{l}\right) \rightarrow \mathcal{H}\left(\underline{G}^{l}\right)$ ) satisfies $\left(f^{*}\right)^{*}=f$ and $f^{*} \cdot g^{*}=(g \cdot f)^{*}$, i.e. it is an algebra anti-involution. We have $e_{\lambda}^{*}=e_{-\lambda}$ and $e_{w}^{*}=e_{w^{-1}}$ in $\mathcal{H}_{-}$. Similarly, $\underline{e}_{\lambda}^{*}=\underline{e}_{-\lambda}$ and $\underline{e}_{w}^{*}=\underline{e}_{w^{-1}}$ in $\mathcal{H}\left(\underline{G}^{l}\right)$.

Theorem 8.3. (i) There is an algebra homomorphism $A: \mathcal{H}\left(\underline{G}^{l}\right) \rightarrow \mathcal{H}_{-}$given by $A\left(\underline{t}_{\lambda}\right)=t_{2 \lambda}$ and $A\left(\underline{e}_{w}\right)=e_{w}$ for $\lambda \in \underline{\Lambda}_{c}$ and $w \in W$. $\mathcal{H}_{-}$.

(ii) The algebra isomorphism $A$ commutes with anti-involutions $*$ on $\mathcal{H}\left(\underline{G}^{l}\right)$ and

Proof. Part (i) follows by comparing relations in $\mathcal{H}\left(\underline{G^{\prime}}\right)$ in $[\mathrm{Lu}]$ and those for $\mathcal{H}_{-}$in Theorem 8.2 For (ii) we first have $A\left(\underline{e}_{w}^{*}\right)=A\left(\underline{e}_{w^{-1}}\right)=e_{w^{-1}}=e_{w}^{*}$ for any $w$ in $W$. By the decomposition $\mathcal{H}_{-}=H \cdot \mathcal{L} \cdot H$, it remains to show that $A\left(\underline{e}_{\lambda}^{*}\right)=\left(A\left(\underline{e}_{\lambda}\right)\right)^{*}$ for a dominant co-character $\lambda$. To that end, let $w$ be the unique element in $W$ such that $w(\Delta)=-\Delta$. Then $\mu=-\lambda^{w}$ is again-dominant. Since

$$
\left\{\begin{array}{l}
\ell(\mu w)=\ell(\mu)+\ell(w) \\
\ell(-w \lambda)=\ell(w)+\ell(-\lambda)
\end{array}\right.
$$

we have $\underline{e}_{w} \underline{e}_{-\lambda}=\underline{e}_{-w \lambda}=\underline{e}_{\mu} \underline{e}_{w}$, and a similar statement exists for elements in $\mathcal{H}_{-}$. Now we have $A\left(\underline{e}_{\lambda}^{*}\right)=A\left(\underline{e}_{-\lambda}\right)=A\left(\underline{e}_{w}^{-1} \underline{e}_{\mu} \underline{e}_{w}\right)=e_{w}^{-1} A\left(\underline{e}_{\mu}\right) e_{w}=p^{-\ell(\mu) / 2} e_{w}^{-1} e_{2 \mu} e_{w}=$ $p^{-\ell(\mu) / 2} e_{-2 \lambda}=p^{-\ell(\lambda) / 2} e_{2 \lambda}^{*}=A\left(\underline{e}_{\lambda}\right)^{*}$, as required. 


\section{REPRESENTATIONS WITH IWAHORI FIXED VECTORS}

Let $I$ and $I^{\prime}$ denote the Iwahori subgroups of $G$ and $\underline{G}^{l}$, respectively, which give rise to isomorphic Iwahori Hecke algebras $\mathcal{H}_{-}$and $\mathcal{H}=\mathcal{H}\left(\underline{G}^{l}\right)$ in Theorem 8.3. Let $\mathcal{R}\left(\mathcal{H}_{-}\right)$and $\mathcal{R}(\mathcal{H})$ denote the categories of finite dimensional representations of the Iwahori-Hecke algebras $\mathcal{H}_{-}$and $\mathcal{H}$, respectively.

Let $\mathcal{R}_{-}^{I}(G)$ denote the category of admissible smooth genuine representations $V$ of $G$ such that $V^{I}$ generates $V$ as a $G$-module. Similarly we let $\mathcal{R}^{I^{\prime}}\left(\underline{G}^{l}\right)$ denote the category of admissible smooth representations $V$ of $\underline{G}^{l}$ such that $V^{I^{\prime}}$ generates $V$ as a $\underline{G}^{l}$-module.

By $\mathrm{BO}$ and $\mathrm{BZ}$, the functor $V \mapsto V^{I^{\prime}}$ is an equivalence of categories from $\mathcal{R}^{I^{\prime}}\left(\underline{G}^{l}\right)$ to $\mathcal{R}(\mathcal{H})$. Let $C_{c}\left(\underline{G}^{l} / I^{\prime}\right)$ denote locally constant, compactly supported, complex valued functions on $\underline{G}^{l} / I^{\prime}$. This is a right $\mathcal{H}$-module. Then the inverse functor is given by $E \mapsto \mathrm{I}(E):=C_{c}\left(\underline{G}^{l} / I^{\prime}\right) \otimes_{\mathcal{H}} E$.

Similarly the functor $V \mapsto V^{I}$ is an equivalence of categories from $\mathcal{R}_{-}^{I}(G)$ to $\mathcal{R}\left(\mathcal{H}_{-}\right)$. Let $C_{c,-}(G / I)$ denote locally constant, compactly supported, complex valued functions on $G / I$ such that $f(\epsilon x I)=\epsilon f(x I)$ for $\epsilon \in \mu_{2}, x \in G$. This is a right $\mathcal{H}_{-}$-module. Then the inverse functor is given by $E \mapsto \mathrm{I}(E):=C_{c,-}(G / I) \otimes_{\mathcal{H}_{-}} E$.

We recall the isomorphism $A: \mathcal{H} \rightarrow \mathcal{H}_{-}$in Theorem 8.3. This establishes an equivalence of categories between $\mathcal{R}(\mathcal{H})$ and $\mathcal{R}\left(\mathcal{H}_{-}\right)$. Hence the following four categories are equivalent:

$$
\mathcal{R}^{I^{\prime}}\left(\underline{G}^{l}\right) \simeq \mathcal{R}(\mathcal{H}) \simeq \mathcal{R}\left(\mathcal{H}_{-}\right) \simeq \mathcal{R}_{-}^{I}(G) .
$$

Suppose $V$ is a representation in $\mathcal{R}_{-}^{I}(G)$; then we call the corresponding representation in $\mathcal{R}^{I^{\prime}}\left(\underline{G}^{l}\right)$ the local Shimura lift of $V$. For example, the Shimura lift of $\Theta\left(\gamma_{p}\right)$ is the trivial representation.

Hermitian representations. We gather some facts from BM1 and BM2]. Let $(\pi, E)$ be a finite dimensional representation of $\mathcal{H}$. We say that $E$ is a Hermitian representation of $\mathcal{H}$ if there exists a Hermitian form $\langle$,$\rangle on E$ such that

$$
\left\langle\pi(f) v_{1}, v_{2}\right\rangle=\left\langle v_{1}, \pi\left(f^{*}\right) v_{2}\right\rangle
$$

for all $v_{1}, v_{2} \in E$ and $f \in \mathcal{H}$. We say that $E$ is a unitary representation of $\mathcal{H}$ if the Hermitian form is positive definite. Similarly, we define Hermitian representations and unitary representations of $\mathcal{H}_{-}$.

Let $V$ be a representation in $\mathcal{R}^{I^{\prime}}\left(\underline{G}^{l}\right)$ (resp. $\mathcal{R}_{-}^{I}(G)$ ). Suppose $\langle$,$\rangle is a non-$ degenerate $\underline{G}^{l}$-invariant (resp. $G$-invariant) Hermitian form on $V$. Then the restriction of the Hermitian form on $V^{I}$ gives a Hermitian representation of $\mathcal{H}$ (resp. $\mathcal{H}_{-}$). Similarly, a unitary representation $V$ gives rise to a unitary representation of the Iwahori-Hecke algebra $\mathcal{H}$ (resp. $\left.\mathcal{H}_{-}\right)$.

Conversely, if $E$ is a Hermitian representation of $\mathcal{H}\left(\right.$ resp. $\left.\mathcal{H}_{-}\right)$, then $\mathrm{I}(E)$ exhibits an $\underline{G}^{l}$-invariant (resp. $G$-invariant) Hermitian form. Moreover, if $E$ is a unitary representation of $\mathcal{H}$, then $\mathrm{I}(E)$ is a unitary representation of $\underline{G}^{l}$. This nontrivial statement is due to Barbasch and Moy (see [BM1] and Thm 8.1 in [BM2]). This, combined with the equivalence of the four categories in (6) (with the middle isomorphism preserving the anti-involution $*$ ), gives:

Theorem 9.1. If $V$ is an irreducible unitary representation in $\mathcal{R}_{-}^{I}(G)$, then its local Shimura lift to $\underline{G}^{l}\left(\mathbb{Q}_{p}\right)$ is unitary. 
Note that the Shimura lift of the exceptional representation $\Theta\left(\gamma_{p}\right)$ is the trivial representation of $\underline{G}^{l}\left(\mathbb{Q}_{p}\right)$. We have proved unitarizability of $\Theta\left(\gamma_{p}\right)$ by global methods.

Corollary 9.2. Assume that $\underline{G} \neq \mathrm{SL}_{2}$. Then the unitary representation $\Theta\left(\gamma_{p}\right)$ is isolated in the unitary dual $G\left(\mathbb{Q}_{p}\right)$.

Proof. Recall that the space of (equivalence classes of) smooth irreducible representations of $G\left(\mathbb{Q}_{p}\right)$ is equipped with a Fell topology [Ta. To every irreducible representation $\Pi$ we can attach a point in the support $\Omega$ of the Bernstein center of $G\left(\mathbb{Q}_{p}\right)$. (The support is a disjoint union of complex varieties of dimension less than or equal to the rank of $G\left(\mathbb{Q}_{p}\right)$.) Tadić in Ta], Theorem 5.7, shows that this map is continuous and closed. Thus, the question as to whether $\Theta_{p}$ is isolated with respect to Fell's topology is equivalent to the same question for the Bernstein center. Since our isomorphism of Hecke algebras gives an equivalence of categories, $\Theta_{p}$ must be isolated in the unitary dual since the trivial representation in the unitary dual of $\underline{G}^{l}\left(\mathbb{Q}_{p}\right)$ is isolated.

Remark. Theorem 9.1 completes a part of $\mathrm{Hu}$. Indeed, a key to Theorem 9.1 is that the isomorphism of Hecke algebras preserves $*$-structures. This was claimed but not verified in $[\mathrm{Hu}$. In retrospect, a verification of this statement at that time was impossible since normalizations of Hecke operators were not properly defined in Sa1].

\section{ACKNOWLEDGMENT}

This work has been motivated by the pioneering works of Gelbart and Sally GS and of Kazhdan and Patterson $[\mathrm{KP}$ on this subject. We would like to thank Dan Ciubotaru, Goran Muić and Peter Trapa for their help and interest in this work. The first author would like to thank the hospitality of the University of Utah while part of this paper was written. The second author is supported by an NSF grant DMS-0551846.

\section{REFERENCES}

[A-V] J. Adam, D. Barbasch, A. Paul, P. Trapa and D. Vogan, Unitary Shimura correspondences for split real groups. JAMS 20 (2007), 701-751. MR2291917(2008i:22008)

[BM1] D. Barbasch and A. Moy, A unitary criterion for p-adic groups. Invent. Math. 98 (1989), 19-37. MR $1010153(90 \mathrm{~m}: 22038)$

[BM2] D. Barbasch and A. Moy, Reduction to real infinitesimal character in affine Hecke algebras. JAMS 6 (1993), 611-635. MR.1186959 (93k:22015)

[BZ] I. N. Bernstein and A. V. Zelevinsky, Induced representations of reductive p-adic groups. I. Ann. Sci. ÉNS (4) 10 (1977), no. 4, 441-472. MR0579172 (58:28310)

[Bo] A. Borel, Admissible representations of a semi-simple group over a local field with vectors fixed under an Iwahori subgroup. Invent. Math. 35 (1976), 233-259. MR0444849 (56:3196)

[Bou] N. Bourbaki, Elements de Mathematique, Algebre, Chapter 8. Hermann, Paris (1958).

[BFG] D. Bump, S. Friedberg and D. Ginzburg Small representations for odd orthogonal groups. IMRN (2003), 1363-1393. MR1968295 (2004c:22022)

[GS] S. Gelbart and P. Sally, Intertwining operators and automorphic forms for the metaplectic group. Proc. Nat. Acad. Sci. U.S.A. 72 (1975), 1406-1410. MR0382175 (52:3063)

[Gr] R. Griess, Schur multipliers of the known finite simple groups. Bulletin of the AMS 78, no. 1 (1972), 68-71. MR0289635 (44:6823)

[Hu] J.-S. Huang, Metaplectic correspondences and unitary representations. Comp. Math. 80, no. 3 (1991), 309-322. MR1134258 (93d:22021) 
[J] H. Jacquet, On the residual spectrum of GL(n). Lie group representations II. LNM 1041 Springer Verlag (1983), 185-208. MR748508 (85k:22045)

[KP] D. A. Kazhdan and S. J. Patterson. Metaplectic forms. Pub. Math. IHES 59 (1984), 35-142. MR.743816 (85g:22033)

[Kn] A. W. Knapp, Nilpotent orbits and some small unitary representations of indefinite orthogonal groups. J. of Funct. Anal. 209 (2004), 36-100. MR2039217(2005h:22018)

[LS] H. Y. Loke and G. Savin, The smallest representation of non-linear covers of odd orthogonal groups. Amer. J. Math. 130 (2008), 763-798. MR2418927 (2009d:20107)

[Lu] G. Lusztig, Affine Hecke algebras and their graded version. JAMS 2 (1989), 599-685. MR.991016 (90e:16049)

[Ma] H. Matsumoto, Sur les sous-groupes arithmétiques des groupes semi-simples déployés. Ann. Sci. ÉNS (4) 2 (1969), no. 1, 1-62. MR0240214 (39:1566)

[MW] C. Møglin and J.-L. Waldspurger, Spectral decomposition and Eisenstein series. Cambridge Tracts in Mathematics, 113. Cambridge University Press, Cambridge (1995). MR 1361168 (97d:11083)

[Mo] C. C. Moore, Group extensions of p-adic and adelic linear groups. Pub. Math. IHES 35 (1968), 5-70. MR0244258 (39:5575)

[Rao] R. R. Rao, On some explicit formulas in the theory of Weil representation. Pacific J. Math. 157, no. 2 (1993), 335-371. MR.1197062 (94a:22037)

[Ro] F. Rodier, Décomposition de la série principale de groupe réductifs p-adiques. LNM 880, Springer-Verlag (1981), 408-424. MR644842 (83i:22029)

[Sa1] G. Savin, Local Shimura correspondence. Math. Ann. 280 (1998), 185-190. MR.929534 (89h:22018)

[Sa2] G. Savin, On ramified representations of covering groups. J. Reine Angew. Math. 566 (2004), 111-134. MR2039325 (2005a:22014)

[Sh] F. Shahidi, On certain L-functions. Amer. J. Math. 103 (1981), no. 2, 297-355. MR610479 (82i:10030)

[St] R. Steinberg, Lectures on Chevalley groups. Yale University (1968). MR0466335 (57:6215)

[Ta] M. Tadić, Geometry of dual spaces of reductive groups (non-Archimedean case). J. Analyse Math. 51 (1988), 139-181. MR.963153 (90c:22057)

[T] P. Trapa, Some small unipotent representations of indefinite orthogonal groups. J. Funct. Anal. 213 (2004), 290-320. MR2078628 (2006f:22009)

[W] A. Weil, Sur certains groupes d'opérateurs unitaires, Acta Math. 111 (1964), 143-211. MR0165033 (29:2324)

Department of Mathematics, National University of Singapore, 2 Science Drive 2 , SINGAPORE 117543

E-mail address: matlhy@nus.edu.sg

Department of Mathematics, University of Utah, Salt Lake City, Utah 84112

E-mail address: savin@math.utah.edu 